\title{
In Situ Summarization with VTK-m
}

\author{
David Thompson \\ Kitware, Inc. \\ david.thompson@kitware.com \\ Berk Geveci \\ Kitware, Inc. \\ berk.geveci@kitware.com
}

\author{
Sebastien Jourdain \\ Kitware, Inc. \\ sebastien.jourdain@kitware.com \\ Robert Maynard \\ Kitware, Inc. \\ robert.maynard@kitware.com \\ Patrick O'Leary \\ Kitware, Inc. \\ patrick.oleary@kitware.com
}

\author{
Andrew Bauer \\ Kitware, Inc. \\ andy.bauer@kitware.com
}

\author{
Ranga Raju Vatsavai \\ North Carolina State University \\ rrvatsav@ncsu.edu
}

\begin{abstract}
Summarization and compression at current and future scales requires a framework for developing and benchmarking algorithms. We present a framework created by integrating existing, productionready projects and provide timings of two particular algorithms that serve as exemplars for summarization: a wavelet-based data reduction filter and a generator for creating image-like databases of extracted features (isocontours in this case). Both support browserbased, post-hoc, interactive visualization of the summary for decisionmaking. A study of their weak-scaling on a distributed multi-GPU system is included.
\end{abstract}

\section{CCS CONCEPTS}

- Information systems $\rightarrow$ Data analytics; • Computing methodologies $\rightarrow$ Shared memory algorithms; Distributed algorithms; Vector / streaming algorithms; Massively parallel algorithms;

\section{KEYWORDS}

in situ visualization, summarization, data reduction

\section{ACM Reference Format:}

David Thompson, Sebastien Jourdain, Andrew Bauer, Berk Geveci, Robert Maynard, Ranga Raju Vatsavai, and Patrick O'Leary. 2017. In Situ Summarization with VTK-m. In Proceedings of ISAV'17: In Situ Infrastructures for Enabling Extreme-Scale Analysis and Visualization, Denver, CO, USA, November 12-17, 2017 (ISAV'17), 5 pages.

https://doi.org/10.1145/3144769.3144777

\section{INTRODUCTION}

Datasets generated through experimentation and simulation are increasingly large; groups around the world have all invested heavily in developing computers capable of processing these datasets,

Permission to make digital or hard copies of all or part of this work for personal or classroom use is granted without fee provided that copies are not made or distributed for profit or commercial advantage and that copies bear this notice and the full citation on the first page. Copyrights for components of this work owned by others than the author(s) must be honored. Abstracting with credit is permitted. To copy otherwise, or republish, to post on servers or to redistribute to lists, requires prior specific permission and/or a fee. Request permissions from permissions@acm.org.

ISAV'17, November 12-17, 2017, Denver, CO, USA

() 2017 Copyright held by the owner/author(s). Publication rights licensed to Association for Computing Machinery.

ACM ISBN 978-1-4503-5139-3/17/11 . .\$15.00

https://doi.org/10.1145/3144769.3144777 resulting in significant experimentation to determine the most viable architecture. These systems typically include message passing interface (MPI) based clusters with graphic processing unit (GPU) stream processors and many-core central processing units (CPUs). Throughout this paper, we will call these Distributed HeterogeneousCore Systems (DHCSs).

DHCSs are changing how processing is performed; storage and network limits force saved results to smaller and smaller fractions of the data. Because of this, summarization - an irreversible transformation of data into a high-level synopsis - and compression - a reversible or approximately reversible transformation of data into a compact form - are essential for reaching the largest scales.

A key insight in choosing useful summarization and compression strategies is to realize that the primary purpose of analysis and visualization is to inform human decisions. These summarized/compressed results must provide (1) information for decisionmaking as well as (2) information to validate that the processing encountered no significant errors.

We have previously developed techniques that allow advanced simulation codes to scale to a million or more homogeneous computer processes by performing in situ analysis and visualization. To apply in situ summarization techniques to exascale computations, we must scale these algorithms to billions of multi-core, stream, and many-core processes given the current trends in DHCS development.

Our work-in-progress is the investigation of algorithms to support summarization and development of a flyweight in situ summarization infrastructure to perform scalability analysis of these new algorithms on exemplar architectures. In this paper, we present the effectiveness of two summarizations: data reduction and featurebased extracts (as a visual summary). This initial work makes contributions to in situ analysis and visualization as described below.

In Situ Infrastructure. We provide a mini-app plus a flyweight, hybrid environment for instrumenting and benchmarking in situ summarization algorithms on DHCSs. It is built on productionquality software that allows developers to leverage previous work including scalable rendering and data parallel algorithms.

Data Parallel Summarization. Our hybrid in situ summarization infrastructure incorporates a portable framework for implementing data parallel analysis and visualization algorithms on DHCSs.

Sorted Compositing Extracts. By enhancing in situ image extracts, our feature based extracts filter provides a mechanism for analyzing 
results in context using transparency and compositing to create new visual content.

Transform-Based Data Reduction. Our in situ summarization infrastructure minimizes data movement while performing the wavelet transform data reduction on data products residing in memory of the HPC infrastructures. Reducing data movement is the key to decrease time to discover, understand, predict and design through data analysis.

In the sections that follow, we explain how this approach to in situ summarization meets our goals for decision-making.

\section{RELATED WORK}

Our approach has several contributions and, therefore, we review related work in relevant areas. In situ methodologies and infrastructures provide essential tools to reduce the cost of data movement and storage required for post hoc analysis and visualization [8]. MPI-based homogeneous in situ processing research studies dominated the prior work $[9,14,16,17,19,20,26,27,32,35,36]$ leaving few studies examining approaches and performance for hybrid architectures of existing extreme-scale systems and those envisioned in the near future [2].

In Situ Infrastructure. Ayachit et al. [6] presented the SENSEI generic in situ interface, which allows developers to instrument a code once and use any number of in situ methods and infrastructures. In addition, in situ methods written with the SENSEI interface can expect them to run in any number of in situ infrastructures. Our infrastructure leverages the SENSEI generic in situ interface for portability, but we extend this work to support hybrid in situ summarization with a portable data parallel framework. Previous work centered on the batch in situ visualization use case utilizing multi-core, stream and many-core processors [23, 25], while others [18] studied idle time and GPU context switching. Our work builds on these explorations but is focused on summarization use cases to efficiently condense usable information from data residing in memory on the compute nodes of DHCSs.

Data Parallel Summarization. Our hybrid in situ summarization infrastructure incorporates, VTKm [30], a portable framework for implementing data parallel analysis and visualization algorithms on DHCSs. VTKm has utilized research and development of three forerunner projects (PISTON [25], Dax [29] and EAVL [28]) to create a unified framework for analysis and visualization on existing and next-generation DHCSs. Our work utilizes some visualization algorithms in VTKm while adding new summarization methods.

Sorted Compositing Extracts. The Cinema [3,31] approach was proposed to alleviate the challenge [10] concerning a priori definition of rendering and visualization parameters used in a batch mode in situ image generation while providing a selectable level of interactive exploration. Cinema is a generalization of Tikhonova et al.'s [34] "explorable images" and Kageyama and Yamada's [22] "special video player" work, affording greater interactivity for exploration. Our work enhances Cinema in situ image databases using a technique for capturing sorted compositing images enabling the analysis of results in context using transparency and compositing to create new visual content.

Transform-Based Data Reduction. Common data reduction techniques include data deduplication, compression, feature selection

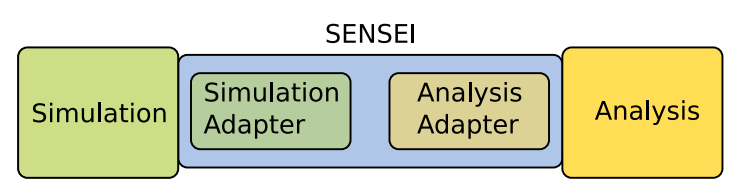

Figure 1: SENSEI's architecture which allows interoperating with a variety of simulations and analyses.

and dimensionality reduction, and sampling [7, 12, 33]. Our prototypes deal with data on a regular grid, which is often compressed by transforming correlated values into a form where the transformed values are independent of each other (i.e., decorrelated). Decorrelating data implies that redundancy is eliminated; a lossy compression can then be obtained by quantizing this decorrelated data. The discrete cosine transformation (DCT) and the wavelet transform [13] are both examples of this approach. Past research and development on parallel wavelet transforms has focused on homogeneous CPU [1] or GPU [4, 15] solution strategies. Recent work leverage VTKm to create a portable data parallel wavelet transform [24]. The scholars responsible for the Li et al. paper [24] argued that these transforms are likely fast enough for in situ use cases. Our research evaluates the performance of a simple 3D Haar wavelet transform using VTKm within our in situ summarization infrastructure.

\section{APPROACH}

Our approach to summarization is to create a benchmarking environment that can leverage DHCS architectures. At the core of this environment are three open-source technologies: SENSEI, a flyweight in situ interface with a common data model; VTKm, a toolkit of scientific analysis and visualization algorithms for emerging processor architectures; and VTK, an open-source, software system for 3D computer graphics, image processing, and visualization.

In the following subsections, we provide more detail on these three open-source technologies and how we utilized them to create the in situ summarization infrastructure.

SENSEI provides a simple API by which simulations and miniapplications can provide direct access to their data for analysis. SENSEI's goal is to provide a "write once, use anywhere" environment which is only achieved using a mutually agreed-upon method for communicating the data between the simulation and the analysis - the data model. Our summarization platform utilizes SENSEI's generic data interface to provide the backbone of the flyweight hybrid in situ summarization infrastructure.

The SENSEI interface (see Figure 1) includes a data adapter to translate between simulation data structures and the SENSEI data model. The analysis adapter performs any required transformations of the data as it passes the results to any analysis method. The in situ bridge merely initializes the data adapter and executes all selected analysis methods invoking the analysis adapter. Because of the simple data model in SENSEI, we are able to write analysis adapters that expose simulation state to VTKm with no data movement.

VTKm provides infrastructure for writing computational kernels so they are portable across DHCS architectures [30]. Kernels are 


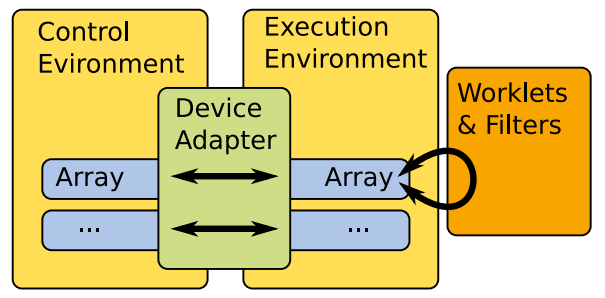

Figure 2: VTKm's architecture lazily transfers arrays to streaming processors for analysis.

scheduled by a control environment and run on arrays in an execution environment (a many-core CPU or a GPU). Arrays passed to each kernel are kept in an execution environment until movement is absolutely required.

To demonstrate accelerated, in situ, summarization, we have implemented two algorithms to illustrate how data reduction and image-based summaries can be accomplished with VTKm:

\subsection{Data reduction}

Given a vector of $\mathrm{N}$ values $x=\left(x_{1}, x_{2}, \ldots, x_{N}\right)$, we approximate them by applying a low-pass filter that halves the original vector size by using a pair-wise average: $s_{k}=\frac{x_{2 k-1}+x_{2 k}}{2}, \forall k \in[1, \ldots, N / 2]$ To recover the original list $\mathrm{x}$, we could create an additional detail vector with a high-pass filter $d_{k}=\frac{x_{2 k}-x_{2 k-1}}{2}, \forall k \in[1, \ldots, N / 2]$. We could then sum the two vectors $s_{k}+d_{k}$ to recover the even entries $x_{2 k}$ and take their difference to obtain odd entries $x_{2 k-1}$. This transformation from $x$ to $(s \mid d)$ is called the Discrete Haar Wavelet (DHW) transformation. To extend this to 3D, simply perform the DHW transform in the $\mathrm{x}$ direction, $\mathrm{y}$ direction, and then $\mathrm{z}$ direction. To perform data reduction, we only need to save the combined $\mathrm{x}, \mathrm{y}$ and $\mathrm{z}$ low-pass result vector as shown in Figure 3a.

As a prototype data reduction algorithm, we use the principle of the 3D DHW transform but keep only the approximate values in each pass to produce a reduced volume where each reduced voxel corresponds to the average value of its eight source voxels. For non-power of two dimensions, we duplicate boundary values for indices outside the local volume to create a valid average.

\subsection{Visual summarization}

Another technique for summarizing a simulation is a visual summary. Here, we generate ensembles of renderings of the simulation's time history. The renderings created at each step vary camera parameters and illustration techniques (isocontours with adjustable contour values, streamlines, cutting planes, etc.). These renderings include compositing information that allows them to be combined by user interaction after the run, providing a powerful way to explore the summary in much the same way datasets are traditionally examined with general-purpose visualization tools like ParaView [5] and VisIt [11].

The pipeline to generate the renderings must be as efficient as possible. While previous work has used Python scripts, the work for this paper includes a $\mathrm{C}++$ Cinema-database generator. Furthermore, the VTK pipeline has been modified to use VTKm to contour

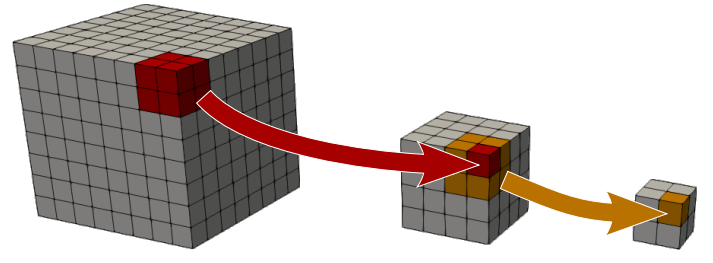

(a)

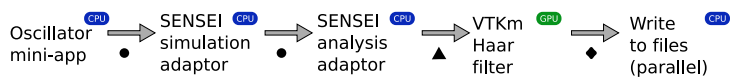

(b)

Figure 3: (a) By running a Haar wavelet filter multiple times, we can reduce the volume to a fixed size that is appropriate for rendering in a browser via ArcticViewer (depicted in Figure 5b). (b) Our wavelet-based reduction pipeline uses VTKm to compute multiple passes of the DHW transform. Circular dots indicate a zero-copy handoff of data. The triangle and diamond indicate where VTKm and VTK transfer data to/from the GPU, respectively.

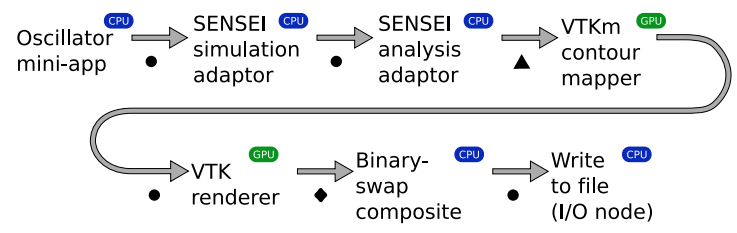

Figure 4: Our visual summary rendering pipeline uses VTKm to compute renderings of isocontours. Circular dots indicate a zero-copy handoff of data. The triangle and diamond indicate where VTKm and VTK transfer data to/from the GPU, respectively.

the data on the GPU and then render it directly. See Figure 4 for an illustration of the pipeline. The modifications we have made are not specific to any simulation and can be generalized from isocontouring to any of the geometric processing done by VTKm.

For the Cinema-like sorted composite image database, we process the images so that they can be composited directly from back to front. This compositing requires us to save an ordering ( 1 byte) and an intensity (1 byte) for each pixel in each layer, allowing re-lighting and other interesting techniques within ArcticViewer [21].

\section{RESULTS}

For our exploration we utilized SENSEI's oscillator mini-application, which is an MPI-based simulator of periodic, damped, or decaying oscillations producing a single scalar field. The simulator convolves each oscillator with a Gaussian kernel of a prescribed width. Inputs are the oscillators, time resolution, duration of the simulation, and the dimensions of the grid. The grid is partitioned between the processes using regular grid 3D domain decomposition. 


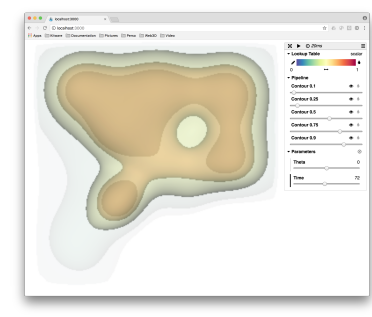

(a)

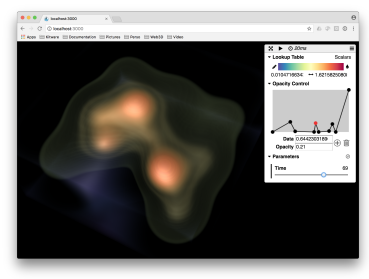

(b)
Figure 5: Complex periodic system composed of a grid of nine big oscillators and four interleaved smaller ones with various frequencies. Visualizations via (a) translucent contours and (b) in-browser volume rendering.

The oscillator mini-application iteratively fills the grid cells with the sum of the convolved oscillator values; the computation on each MPI rank takes $O\left(m N^{3}\right)$ calculations per time step, where $m$ is the number of oscillators and $N$ is the one-dimensional size of the subgrid on the rank. The computation is documented to weak-scale well to more than 50,000 MPI ranks.

Our results were obtained on an SGI ICE X machine named Topaz. Topaz is a heterogeneous system with 3,456 regular nodes, 4 large memory nodes and 32 GPU nodes. We only used the GPU nodes for our tests. Each GPU node has two Intel Xeon E5-2697v3 Haswell processors and one NVIDIA Tesla K40P GPU. The Haswell CPUs have two 14-core processors running at $2.6 \mathrm{GHz}$ and $128 \mathrm{~GB}$ of memory per node. The Tesla K40P has 12 GB of GDDR5 memory.

We ran four cases to characterize our approach:

(1) a baseline simulation where the entire dataset is written at each time step;

(2) a data reduction prototype using repeated Haar filtering to save a fixed-size volume of data per time step appropriate for interactive volume rendering in a web browser;

(3) a visual summary generator using VTK's existing marching cubes and rendering algorithms (on the CPU but with threading enabled) to generate Cinema databases; and

(4) a visual summary generator that uses VTKm to compute contours and render Cinema databases on the GPU.

The first 2 cases illustrate how data reduction can save space while the latter 2 cases illustrate visual summarization. Timings for weak scaling are shown in Table 1.

For cases 1 and 2, the difference in run times is negligible but the output for case 2 is capped at a fixed size per timestep (since we adjust the number of reduction passes as we increase the number of processes per job) while the output for case 1 grows rapidly. For these runs, case 1's output is approximately $523 \mathrm{MB}$ per process per timestep while case 2 is approximately $0.5 \mathrm{MB}$ per timestep. This type of reduction is especially useful for direct numerical simulations of reacting flows since they typically over-resolve spatially in order to capture turbulent flow at the Kolmogorov scale, so the data being reduced varies smoothly over most of the domain. Clearly, as the simulation size is increased, the $\mathrm{I} / \mathrm{O}$ requirements for case 1 are untenable while case 2 produces a summary at a negligible cost.

\begin{tabular}{rrrrr} 
Job size & Case & Run time [s] & Case & Run time [s] \\
\hline 4 & 1 & 326 & 2 & 332 \\
8 & & 338 & & 343 \\
16 & & 335 & & 336 \\
32 & & 328 & & 334 \\
\hline 4 & 3 & 889 & 4 & 984 \\
8 & & 922 & & 998 \\
16 & & 901 & & 1001 \\
32 & & 891 & & 995 \\
\hline
\end{tabular}

Table 1: Timings for weak scaling of summarization and reduction. All runs done with 1 process and 1 GPU per node.

Note that because the oscillator mini-app currently only runs on the CPU, there is a cost for transferring the entire volumetric dataset to the GPU for case 4 while for case 3 only the output contour triangles are transferred to the GPU for rendering. This may account for the small but measurable difference between cases 3 and 4. Our future work involves reworking the simulation to run on the GPU so this can be avoided.

\section{CONCLUSION}

We have demonstrated a framework for in situ summarization that runs algorithms on CPUs and GPUs in a cluster. The premise of summarization is that domain experts can specify the type of summary information that will help them validate the simulation and make a decision; otherwise, the simulation must be repeated to obtain further information.

Our approach is verified with 2 prototype summarizations: a lossy, wavelet-based compression algorithm and a GPU-accelerated, isocontour feature-renderer. Furthermore, we provide a generic framework rather than something tailored to a particular simulation and available at https://ki tware.gi thub.io/summarization/docs.

Future work includes generalizing the prototype VTKm mapper to work with any VTKm filter's output instead of a special case for isocontouring; we intend to accomplish this by allowing filters in VTK which use VTKm to mark arrays held on the GPU so that the mapper can request at render-time that they be bound to OpenGL vertex buffer objects. We also intend to expand the repertoire of VTKm filters available in situ to include other types of summary information such a statistics and function approximations.

\section{ACKNOWLEDGMENTS}

Computer resources to run on Topaz at the U.S. Army Engineer Research and Development Center's DoD Supercomputing Resource Center were provided by the DoD's HPCMP Frontier program. This material is based upon work supported by the U.S. Army Small Business Technology Transfer Program Office and the Army Research Office under Contract Number: W911NF-17-P-0038, program manager Dr. Joseph Myers. The SENSEI enhancements were supported by the Director, Office of Science, Office of Advanced Scientific Computing Research, of the U.S. Department of Energy under Contract No. DE-AC02-05CH11231, through the grant "Scalable Analysis Methods and In Situ Infrastructure for Extreme Scale Knowledge Discovery," program manager Dr. Lucy Nowell. 


\section{REFERENCES}

[1] T. Acharya and P.-S. Tsai. 2004. JPEG2000 Standard for Image Compression: Concepts, Algorithms and VLSI Architectures. Wiley-Interscience.

[2] S. Ahern, A. Shoshani, K.-L. Ma, A. Choudhary, T. Critchlow, V. Pascucci, J. Ahrens, E. W. Bethel, H. Childs, J. Huang, K. Joy, Q. Koziol, G. Lofstead, J. Meredith, K. Moreland, G. Ostrouchov, M. Papka, V. Vishwanath, M. Wolf, N. Wright, and K. Wu. 2011. Scientific Discovery at the Exascale: Report from the DOE ASCR 2011 Workshop on Exascale Data Management, Analysis, and Visualization. Technical Report. Dept. of Energy, Office of Advanced Scientific Computing Research.

[3] J. Ahrens, S. Jourdain, P. O'Leary, J. Patchett, D. H. Rogers, and M. Petersen. 2014 An image-based approach to extreme scale in situ visualization and analysis. In Proceedings of the International Conference for High Performance Computing, Networking, Storage and Analysis. IEEE Press, 424-434.

[4] J. Ao, S. Mitra, and B. Nutter. 2014. Fast and efficient loss-less image compression based on CUDA parallel wavelet tree encoding. In 2014 IEEE Southwest Symposium on Image Analysis and Interpretation (SSIAI). IEEE, 21-24.

[5] U. Ayachit. 2015. The ParaView Guide: A Parallel Visualization Application. Kitware, Inc., Clifton Park, NY.

[6] U. Ayachit, A. Bauer, E. P. N. Duque, G. Eisenhauer, N. Ferrier, J. Gu, K. E. Jansen, B. Loring, Z. Lukic, S. Menon, D. Morozov, P. O'Leary, R. Ranjan, M. Rasquin, C. P. Stone, V. Vishwanath, G. H. Weber, B. Whitlock, M. Wolf, K. J. Wu, and E. W. Bethel. 2016. Performance Analysis, Design Considerations, and Applications of Extreme-scale In Situ Infrastructures. In Proc. of the International Conference for High Performance Computing, Networking, Storage and Analysis (SCâĂŹ16).

[7] T. Ravindra Babu, M. Narasimha Murty, and S.V. Subrahmanya. 2013. Compression Schemes for Mining Large Datasets: A Machine Learning Perspective. Springer Verlag, London.

[8] A. C. Bauer, H. Abbasi, J. Ahrens, H. Childs, B. Geveci, S. Klasky, K.Moreland, P. O'Leary, V. Vishwanath, and E. W. Bethel. 2016. In Situ Methods, Infrastructures, and Applications on High Performance Computing Platforms. Computer Graphics Forum 35, 3 (June 2016)

[9] Wes Bethel, Brian Tierney, Jason lee, Dan Gunter, and Stephen Lau. 2000. Using High-speed WANs and Network Data Caches to Enable Remote and Distributed Visualization. In Supercomputing '00: Proceedings of the 2000 ACM/IEEE conference on Supercomputing. IEEE Computer Society, Dallas, Texas, United States. LBNL45365.

[10] H. Childs. 2007. Architectural challenges and solutions for petascale postprocessing. Fournal of Physics: Conference Series 78, 012012 (2007).

[11] Hank Childs, Eric Brugger, Brad Whitlock, Jeremy Meredith, Sean Ahern, David Pugmire, Kathleen Biagas, Mark Miller, Cyrus Harrison, Gunther H. Weber, Hari Krishnan, Thomas Fogal, Allen Sanderson, Christoph Garth, E. Wes Bethel, David Camp, Oliver Rübel, Marc Durant, Jean M. Favre, and Paul Navrátil. 2012. VisIt An End-User Tool For Visualizing and Analyzing Very Large Data. In High Performance Visualization-Enabling Extreme-Scale Scientific Insight. 357-372.

[12] B. Chizi, L. Rokach, and O. Maimon. 2009. A Survey of Feature Selection Techniques (2 ed.).

[13] I. Daubechies. 1992. Ten Lectures on Wavelets. SIAM, Philadelphia, PA.

[14] Nathan Fabian, Kenneth Moreland, David Thompson, Andrew C. Bauer, Pat Marion, Berk Geveci, Michel Rasquin, and Kenneth E. Jansen. 2011. The ParaView Coprocessing Library: A Scalable, General Purpose In Situ Visualization Library. In IEEE Symposium on Large-Scale Data Analysis and Visualization (LDAV) 2011. Institute of Electrical and Electronics Engineers, 89-96.

[15] A. Garcia and H.-W. Shen. 2005. GPU-based 3D wavelet reconstruction with tileboarding. The Visual Computer 21, 8 (2005), 755-763.

[16] Al Globus. 1995. A Software Model for Visualization of Large Unsteady 3D CFD Results. In 33rd Aerospace Sciences Meeting and Exhibit (AIAA). http: //arc.aiaa.org/doi/abs/10.2514/6.1995-115

[17] T. Goodale, G. Allen, G. Lanfermann, J. Massãş, T. Radke, E. Seidel, and J. Shalf. 2003. The Cactus Framework and Toolkit: Design and Applications. In Vector and Parallel Processing - VECPAR '2002, 5th International Conference. Springer

[18] A. Goswami, Y. Tian, K. Schwan, F. Zheng, J. Young, M. Wolf, G. Eisenhauer, and S. Klasky. 2016. Landrush: Rethinking In-Situ Analysis for GPGPU Workflows. In Proceedings of the 16th IEEE/ACM International Symposium on Cluster, Cloud and Grid Computing (CCGrid). IEEE, Cartagena, Colombia.

[19] Robert Haimes. 1995. Concurrent Distributed Visualization and Steering. In Parallel Computational Fluid Dynamics: Implementations and Results Using Parallel Computers.

[20] Janet Jacobsen, E. Wes Bethel, Akhil Datta-Gupta, and Preston Holland. 1995. Petroleum Reservoir Simulation in a Virtual Environment. In Proceedings of the 13th Symposium on Reservoir Simulation (SPE). San Antonio TX, USA.

[21] S. Jourdain, S. Wittenburg, T. Wright, A. Helser, and P. O'Leary. 2017. ArcticViewer. https://kitware.github.io/arctic-viewer/. (2017). [Online; accessed Aug 29, 2017].

[22] A. Kageyama and T. Yamada. 2014. An approach to exascale visualization: Interactive viewing of in-situ visualization. Computer Physics Communications 185, 1 (2014), 79-85.
[23] Matthew Larsen, Eric Brugger, Hank Childs, Jim Eliot, Kevin Griffin, and Cyrustit Harrison. 2015. Strawman: A Batch In Situ Visualization and Analysis Infrastructure for Multi-Physics Simulation Codes. In Proceedings of the First Workshop on In Situ Infrastructures for Enabling Extreme-Scale Analysis and Visualization.

[24] S. Li, N. Marsaglia, V. Chen, C. Sewell, J. Clyne, and H. Childs. 2017. Achieving Portable Performance For Wavelet Compression Using Data Parallel Primitives. In Eurographics Symposium on Parallel Graphics and Visualization (EGPGV). Barcelona, Spain

[25] L. Lo, C. Sewell, and J. Ahrens. 2012. PISTON: A Portable Cross-Platform Framework for Data-Parallel Visualization Operators. In Proc. Eurographics Symp. Parallel Graphics and Visualization (EGPGV). 11-20.

[26] Jay F Lofstead, Scott Klasky, Karsten Schwan, Norbert Podhorszki, and Chen Jin. 2008. Flexible I/O and integration for scientific codes through the adaptable I/O system (ADIOS). In Proceedings of the 6th international workshop on Challenges of large applications in distributed environments. ACM, 15-24.

[27] K.-L. Ma. 1995. Runtime volume visualization of parallel CFD. In Proceedings of Parallel CFD Conference. 307-314.

[28] J. S. Meredith, S. Ahern, D. Pugmire, and R. Sisneros. 2012. EAVL: The ExtremeScale Analysis and Visualization Library. In Proc. Eurographics Symp. Parallel Graphics and Visualization. 21-30.

[29] K. Moreland, B. King, R. Maynard, and Kwan-Liu Ma. 2012. Flexible Analysis Software for Emerging Architectures. In Proc. SC'12 Companion: High-Performance Computing, Networking Storage and Analysis. 821-826.

[30] K. Moreland, C. Sewell, W. Usher, L.t. Lo, J. Meredith, D. Pugmire, J. Kress, H. Schroots, K.L. Ma, H. Childs, M. Larsen, C.M. Chen, R. Maynard, and B. Geveci. 2016. VTK-m: Accelerating the Visualization Toolkit for Massively Threaded Architectures. 36, 3 (May 2016), 48-58.

[31] P. O'Leary, J. Ahrens, S. Jourdain, S. Wittenburg, D. H. Rogers, and M. Petersen. 2016. Cinema image-based in situ analysis and visualization of MPAS-ocean simulations. Parallel Comput. 55 (2016), 43-48.

[32] Steven G. Parker, Christopher R. Johnson, and David Beazley. 1997. Computational Steering Software Systems and Strategies. IEEE Comput. Sci. Eng. 4, 4 (Oct. 1997), 50-59. https://doi.org/10.1109/99.641609

[33] D. Salomon. 2007. Data Compression: The Complete Reference. Springer Verlag, London.

[34] A. Tikhonova, C. D. Correa, and K.-L. Ma. 2010. Visualization by proxy: A novel framework for deferred interaction with volume data. 16, 6 (Nov. 2010), 1551-1559.

[35] Tiankai Tu, Hongfeng Yu, Leonardo Ramirez-Guzman, Jacobo Bielak, Omar Ghattas, Kwan-Liu Ma, and David R. O'Hallaron. 2006. From Mesh Generation to Scientific Visualization: An End-to-End Approach to Parallel Supercomputing. In Proceedings of the International Conference for High Performance Computing, Networking, Storage and Analysis. DOI 10.1109/SC.2006.32.

[36] Brad Whitlock, Jean M Favre, and Jeremy S Meredith. 2011. Parallel in situ coupling of simulation with a fully featured visualization system. In Proceedings of the 11th Eurographics conference on Parallel Graphics and Visualization. Eurographics Association, 101-109. 